\title{
Aminolysis of $N$-Tritylamino Acid Benzotriazolyl Esters with Concentrated Aqueous Amines and its Applications in Synthesis
}

\author{
Petros Mamos, ${ }^{a}$ Elias Dalatsis, ${ }^{\mathrm{b}}$ Costas Athanassopoulos, ${ }^{\mathrm{b}}$ Georgios Balayiannis, \\ Dionissios Papaioannou ${ }^{\mathrm{b}, *}$ and George W. Francis ${ }^{\mathrm{c}}$ \\ a Department of Medicine, University of Patras, 26500 Patras, Greece, ${ }^{b}$ Department of Chemistry, University of
Patras, 26500 Patras, Greece and ' Department of Chemistry, University of Bergen, Allegaten $41, \mathrm{~N}-4007$ Bergen,
Norway
}

\begin{abstract}
Mamos, P., Dalatsis, E., Athanassopoulos, C., Balayiannis, G., Papaioannou, D. and Francis, G. W., 1998. Aminolysis of $N$-Tritylamino Acid Benzotriazolyl Esters with Concentrated Aqueous Amines and its Applications in Synthesis. Acta Chem. Scand. 52: 227-231. (C) Acta Chemica Scandinavica 1998.

Aminolysis of $(S)$ - $N$-tritylamino acid benzotriazolyl esters with concentrated aqueous amines produces high yields of the corresponding amides which can be readily detritylated with $p$-toluensulfonic acid or reduced with lithium aluminium hydride to provide chiral ethane-1,2-diamine derivatives.
\end{abstract}

A variety of biologically important peptides occur in Nature in the form of $C$-terminal amides. Such peptide amides can be prepared in solution without racemization by direct amidation of the corresponding peptide acids or by amidation of suitably protected $C$-terminal amino acids followed by assembly of the peptide chain to the required length. ${ }^{1}$ We have reported that dicyclohexylcarbodiimide (DCC)-mediated activation of $\mathrm{N}$-tritylamino acids with 1-hydroxybenzotriazole (HOBt) produces the corresponding readily isolable esters $\mathbf{1}$ as an equilibrium mixture of the 'active' ester (1-I) and 'active' amide (1-II) forms. ${ }^{2}$ These esters are highly stable to hydrolysis reactions, are resistant to racemization, ${ }^{3}$ and show potent acylating abilities and thus constitute valuable intermediates in peptide synthesis. ${ }^{4}$ We now wish to report that esters 1 can be employed in the simple and convenient synthesis of amides using cheap, commercially available, concentrated aqueous (aq.) solutions of simple amines. Others have reported that unrefined preparations of such active esters, $\mathrm{N}$-protected with groups of the urethane type, may be directly converted into primary amides by the action of ammonium hydroxide. ${ }^{5}$

As esters 1 are extremely hydrophobic and exhibit their acylating potential in concentrated solutions, the use of the most concentrated commercially available aq. solutions of amines for amidation is mandatory. Thus, treatment of concentrated solutions of esters 1 in DMF with $30 \%$ aq. $\mathrm{NH}_{3}, 40 \%$ aq. $\mathrm{MeNH}_{2}, 33 \%$ aq. $\mathrm{EtNH}_{2}$ or $40 \%$ aq. $\mathrm{Me}_{2} \mathrm{NH}$ for less than $30 \mathrm{~min}$ at $0{ }^{\circ} \mathrm{C}$ in most cases produced cleanly (TLC), the corresponding crystal-

\footnotetext{
* To whom correspondence should be addressed.
}

line amides 2 in 80-91\% yields (Table 1). No sign of hydrolysis of the active esters was detected (TLC) in the amidation reaction mixtures. In accord with earlier observations, ${ }^{2}$ the ester form (1-I) reacts much faster than the amide form (1-II). The latter is rapidly formed in solution when using pure ester form preparations for amidation. In cases where extreme steric hindrance is encountered, e.g., when using Trt-Val-OBt (1b) for amidation, small amounts of the amide form remained unreacted on treatment with both $\mathrm{MeNH}_{2}$ and $\mathrm{Me}_{2} \mathrm{NH}$ aq. solutions, and were identified as such after isolation by flash column chromatography (FCC) and characterisation by IR and MS (see Experimental). The present method is simple and convenient as it avoids the use of these amines either in their gaseous form or as their corresponding commercially available, but hygroscopic, hydrochlorides.

The trityl (Trt) protective group can be readily removed on brief treatment with $\mathrm{TsOH} \cdot \mathrm{H}_{2} \mathrm{O}$ in refluxing isopropyl alcohol, a fact demonstrated by the preparation of the tosylates $\mathbf{3 a}-\mathbf{c}$, in $75-95 \%$ yields. Tosylates like $\mathbf{3}$ can of course be routinely used for the preparation of analogues of biologically important peptides for structure-activity relationship studies. Furthermore, taking into consideration the compatibility of the Trt group with complex metal hydrides, ${ }^{6}$ a property not shared by the protecting groups of the urethane type commonly used for $N^{\alpha}$-protection of amino acids, amides such as $\mathbf{2}$ were postulated as convenient sources of chiral ethane1,2-diamines. In point of fact, treatment of concentrated solutions of, e.g., the amides $\mathbf{2 a}, \mathbf{b}, \mathbf{g}$ and $\mathbf{m}$ with $\mathrm{LiAlH}_{4}$ 
<smiles>[Z17]N[C@H]([R])C(=O)On1nnc2ccccc21</smiles><smiles>[X]C([Z])=C1CCCN1</smiles>

If $\mathrm{Z}=\mathrm{O}, \mathrm{X}=\mathrm{OBt}$ 2i $Z=O, X=N H M e$ 4b $\mathrm{Z}=\mathrm{H}_{2}, \mathrm{X}=\mathrm{NHMe}$<smiles>[Z]C(N[Tl])C(=O)O</smiles><smiles>[R]C(CN([R])[18F])NC</smiles>

4a $\mathrm{R}=\mathrm{Me}, \mathrm{R}^{\prime}=\mathrm{H}^{\prime}$

4c $\mathrm{R}=\mathrm{CH}_{2} \mathrm{Ph}, \mathrm{R}^{\prime}=\mathrm{H}$

4d $\mathrm{R}=\left(\mathrm{CH}_{2}\right)_{3} \mathrm{OH}, \mathrm{R}^{\prime}=\mathrm{Me}$

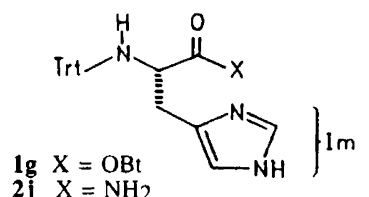
$2 \mathrm{j} X=\mathrm{NH}_{2}$

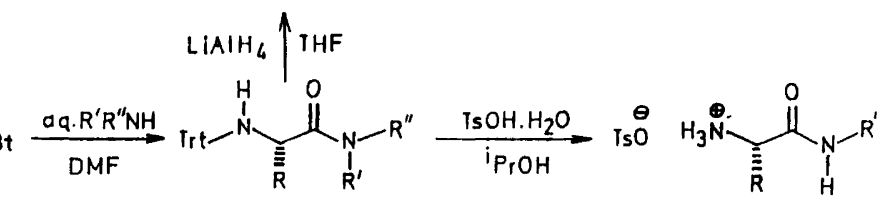

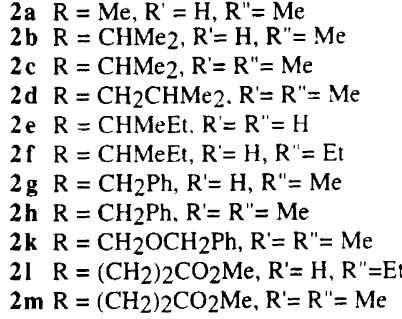

Fig. 1. Synthetic outline and structures for compounds discussed in the present work.

Table 1. Yields and physical data of $\alpha$-amino amide and ethane-1,2-diamine derivatives. ${ }^{a}$

\begin{tabular}{|c|c|c|c|c|}
\hline Compound & Yield (\%) & M.p. $/{ }^{\circ} \mathrm{C}$ & {$[\alpha]_{D}^{28}$} & $\mathrm{IR} \mathrm{v} / \mathrm{cm}^{-1}$ \\
\hline Trt-Ala-NHMe (2a) & 82 & $167-168$ & +32.8 & $3300,1664,1652$ \\
\hline Trt-Val-NHMe (2b) & 88 & 190-191 & +75.1 & 3316,1640 \\
\hline Trt-Val-NMe ${ }_{2}(2 \mathrm{c})$ & 80 & $111-112$ & +107.8 & $3296,1646,1634$ \\
\hline Trt-Leu-NMe ${ }_{2}$ (2d) & 82 & $105-106$ & +95.4 & $3308,1640,1628$ \\
\hline Trt-lle- $\mathrm{NH}_{2}(2 \mathrm{e})$ & 87 & $164-165$ & +33.4 & $3452,3302,3184,1682,1652,1611$ \\
\hline Trt-Ile-NHEt (2f) & 84 & $168-170$ & +21.1 & 3332,1640 \\
\hline Trt-Phe-NHMe (2g) & 91 & $168-169$ & +21.7 & $3284,1682,1650$ \\
\hline Trt-Phe-NMe 2 (2h) & 82 & $145-146$ & +121.4 & $3307,1644,1632$ \\
\hline Trt-Pro-NHMe (2i) & 80 & $134-135$ & -73.3 & $3356,1655,1642$ \\
\hline Trt-His- $\mathrm{NH}_{2}(2 \mathrm{j})$ & 81 & $121-123$ & +35.9 & $3446,3305,3234,1674$ \\
\hline Trt-Ser(Bzl)-NMe ${ }_{2}(\mathbf{2 k})$ & 81 & $152-153$ & +144.9 & 3286,1632 \\
\hline Trt-Glu(Me)-NHEt (2l) & 84 & $164-165$ & +56.4 & $3306,1732,1634$ \\
\hline Trt-Glu(Me)-NMe $2(2 m)$ & 85 & $138-139$ & +74.3 & $3292,1738,1636,1626$ \\
\hline TsOH·H-Ala-NHMe (3a) & 89 & $145-146$ & +13.7 & $3328,1694,1684^{b}$ \\
\hline $\mathrm{TsOH} \cdot \mathrm{H}-\mathrm{Ph}$-NHMe (3b) & 90 & $192-193$ & $+33.4^{c}$ & 3328,1662 \\
\hline $\mathrm{TsOH} \cdot \mathrm{H}-\mathrm{His}-\mathrm{NH}_{2}(3 \mathrm{c})$ & 75 & $225-230$ & -4.5 & $3428,1688,1678$ \\
\hline $4 a$ & 60 & $180-182$ & +26.3 & 3295,3210 \\
\hline $4 b$ & 65 & $140-141$ & -31.0 & 3415,3268 \\
\hline 4c & 74 & Oil & +49.2 & 3286 \\
\hline 4d & 88 & Oil & +63.3 & 3346,3284 \\
\hline
\end{tabular}

${ }^{a}$ All compounds gave satisfactory microanalytical data $(\mathrm{C}, \mathrm{H})$ within \pm 0.3 of the calculated values. Yields of reactions are not optimized. Optical rotation values were obtained for $1 \%$ solutions in DMF unless otherwise stated. ${ }^{b}$ Tosylates showed in addition a broad band at $3200-2500 \mathrm{~cm}^{-1}$. ${ }^{c} 1 \%$ in $\mathrm{MeOH}$.

in refluxing THF, usually for $1-2 \mathrm{~d}$, produced $60-88 \%$ yields of the corresponding diamine derivatives $\mathbf{4 a - d}$. Worth noting is the reduction of amide $2 \mathrm{~m}$ which pro- duced the diamino alcohol $\mathbf{4 d}$ in less than $1 \mathrm{~h}$, a result which may be attributed to the participation of the newly formed hydroxy function from the reduction of the 
Table 2. El-MS and $200 \mathrm{M} \mathrm{Hz}{ }^{1} \mathrm{H}$ NMR data of $\alpha$-tritylamino amides. ${ }^{a}$

\begin{tabular}{|c|c|c|}
\hline Amide & MS data & NMR data $(\delta, \mathrm{ppm})$ \\
\hline $2 a$ & $344(M), 286(M-\mathrm{CONHMe})$ & $\begin{array}{l}6.538(1 \mathrm{H}, \text { br q, J } 5.00 \mathrm{~Hz}, \mathrm{CONH}), \\
3.281\left(1 \mathrm{H}, \mathrm{q}, J 6.97 \mathrm{~Hz},{ }^{*} \mathrm{CH}\right), 2.504(1 \mathrm{H}, \mathrm{br}, \mathrm{TrtNH}), \\
2.481\left(3 \mathrm{H}, \mathrm{d}, J 5.00 \mathrm{~Hz}, \mathrm{NHCH}_{3}\right), \\
1.153\left(3 \mathrm{H}, \mathrm{d}, J 6.97 \mathrm{~Hz}, \mathrm{CHCH}_{3}\right)\end{array}$ \\
\hline $2 \mathbf{b}$ & $372(M), 314(M-$ CONHMe $)$ & $\begin{array}{l}5.877(1 \mathrm{H}, \text { br q, J } 4.85 \mathrm{~Hz}, \mathrm{CONH}), 3.092\left(1 \mathrm{H}, \mathrm{br},{ }^{*} \mathrm{CH}\right), \\
2.283(3 \mathrm{H}, \mathrm{d}, \mathrm{J} 4.85 \mathrm{~Hz}, \mathrm{NHCH}), \\
2.722(1 \mathrm{H}, \mathrm{br}, \mathrm{TrtNH}) 1.890(1 \mathrm{H}, \mathrm{m}, \mathrm{CHMe})^{\prime}, \\
0.942 \text { and } 0.932(6 \mathrm{H}, \text { two d, J } 6.94 \mathrm{~Hz}, g e m-\mathrm{Me})\end{array}$ \\
\hline 2c & $343\left(M-\mathrm{Me}_{2} \mathrm{CH}\right), 314(\mathrm{M}-\mathrm{CONMe})$ & $\begin{array}{l}3.260(1 \mathrm{H}, \mathrm{br} \mathrm{d}, \operatorname{TrtNH}), 3.442\left(1 \mathrm{H}, \mathrm{m},{ }^{*} \mathrm{CH}\right), \\
2.373\left(3 \mathrm{H}, \mathrm{s}, \mathrm{NCH}_{3}\right), 2.428\left(3 \mathrm{H}, \mathrm{s}, \mathrm{NCH}_{3}\right), \\
2.090(1 \mathrm{H}, \mathrm{m}, \mathrm{CHMe}) \\
1.061(3 \mathrm{H}, \mathrm{d}, \mathrm{J} 6.91 \mathrm{~Hz}, \text { gem-Me }), \\
0.967(3 \mathrm{H}, \mathrm{d}, \mathrm{J} 6.91 \mathrm{~Hz}, \text { gem-Me) }\end{array}$ \\
\hline $2 d$ & $400(M), 342\left(M-\mathrm{CONMe}_{2}\right)$ & $\begin{array}{l}3.436(1 \mathrm{H}, \mathrm{br}, \mathrm{TrtNH}), 3.279\left(1 \mathrm{H}, \mathrm{br},{ }^{*} \mathrm{CH}\right), \\
2.474\left(3 \mathrm{H}, \mathrm{s}, \mathrm{NCH}_{3}\right), 2.381\left(3 \mathrm{H}, \mathrm{s}, \mathrm{NCH}_{3}\right), \\
2.1-1.9\left(3 \mathrm{H}, \mathrm{m}, \mathrm{CH}_{2} \text { and } \mathrm{CHMe}\right) \\
0.942(6 \mathrm{H}, \mathrm{d}, \mathrm{J} 6.93 \mathrm{~Hz}, \text { gem-Me })\end{array}$ \\
\hline $2 \mathbf{e}$ & $328\left(M-\mathrm{CONH}_{2}\right), 86\left[\mathrm{EtC}(\mathrm{Me}) \mathrm{HNH}_{2}\right]$ & $\begin{array}{l}3.212\left(1 \mathrm{H}, \mathrm{br},{ }^{*} \mathrm{CH}\right), 2.661(1 \mathrm{H}, \mathrm{br}, \mathrm{TrtNH}), \\
1.611(1 \mathrm{H}, \mathrm{br}, \mathrm{CHMe}), 1.281\left(2 \mathrm{H}, \mathrm{m}, \mathrm{CH} \mathrm{H}_{2} \mathrm{Me}\right), \\
0.903\left(3 \mathrm{H}, \mathrm{d}, \mathrm{J} 6.36 \mathrm{~Hz}, \mathrm{CH}_{3} \mathrm{CH}\right), \\
0.723\left(3 \mathrm{H}, \mathrm{t}, \mathrm{J} 7.17 \mathrm{~Hz}, \mathrm{CH}_{3} \mathrm{CH}_{2}\right)\end{array}$ \\
\hline $2 f$ & $\begin{array}{l}328(\mathrm{M}-\mathrm{CONHEt}), 258(\text { TrtNH }) \\
86\left[\mathrm{EtCH}(\mathrm{Me}) \mathrm{NH}_{2}\right]\end{array}$ & $\begin{array}{l}6.138(1 \mathrm{H}, \text { br t }, J 5.6 \mathrm{~Hz}, \mathrm{CONH}), \\
3.173\left(1 \mathrm{H}, \mathrm{dd}, J 3.13 \text { and } 4.96 \mathrm{~Hz},{ }^{*} \mathrm{CH}\right) \text {, } \\
2.852\left(2 \mathrm{H}, \mathrm{m}, \mathrm{NCH}_{2}\right), 2.630(1 \mathrm{H}, \mathrm{d}, J 5.06 \mathrm{~Hz}, \mathrm{TrtNH}), \\
1.54-1.06\left(3 \mathrm{H}, \mathrm{m}, \mathrm{MeCH}+\mathrm{MeCH}_{2}\right), \\
0.898\left(3 \mathrm{H}, \mathrm{t}, J 7.32 \mathrm{~Hz}, \mathrm{NCH}_{2} \mathrm{CH}_{3}\right), \\
0.880\left(3 \mathrm{H}, \mathrm{d}, J 7.28 \mathrm{~Hz}, \mathrm{CHCH}_{3}\right) \\
0.746\left(3 \mathrm{H}, \mathrm{t}, J 7.05 \mathrm{~Hz}, \mathrm{CH}_{2} \mathrm{CH}_{2}\right)\end{array}$ \\
\hline $2 g$ & $\begin{array}{l}362(\mathrm{M}-\mathrm{CONHMe}) \\
343(\mathrm{M}-\mathrm{Ph}), 329\left(\mathrm{M}-\mathrm{PhCH}_{2}\right) \\
120\left(\mathrm{PhCH}_{2} \mathrm{CHNH}_{2}\right)\end{array}$ & 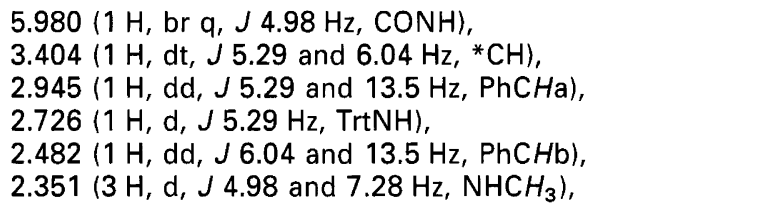 \\
\hline $2 \mathrm{~h}$ & $\begin{array}{l}435(\mathrm{M}+\mathrm{H}), 362\left(\mathrm{M}-\mathrm{CONMe}_{2}\right) \\
357(\mathrm{M}-\mathrm{Ph}), 343\left(\mathrm{M}-\mathrm{PhCH}_{2}\right) \\
120\left(\mathrm{PhCH}_{2} \mathrm{CHNH}_{2}\right), 91\left(\mathrm{PhCH}_{2}\right) \\
72\left(\mathrm{CONMe}_{2}\right)\end{array}$ & $\begin{array}{l}3.618(1 \mathrm{H}, \mathrm{br}, \mathrm{TrtNH}), 3.429\left(1 \mathrm{H}, \mathrm{br},{ }^{*} \mathrm{CH}\right) \\
3.150(1 \mathrm{H}, \mathrm{dd}, J 5.53 \text { and } 13.03 \mathrm{~Hz}, \mathrm{PhCHa}) \text {, } \\
2.870\left(1 \mathrm{H}, \mathrm{dd}, J 8.90 \text { and } 12.83 \mathrm{~Hz}, \mathrm{PhCHb}^{\prime},\right. \\
2.207\left(3 \mathrm{H}, \mathrm{s}, \mathrm{NCH}_{3}\right), 1.758\left(3 \mathrm{H}, \mathrm{s}, \mathrm{NCH}_{3}\right)\end{array}$ \\
\hline $2 \mathbf{i}$ & $70(\mathrm{M}-\mathrm{Trt}-\mathrm{MeNCO})$ & 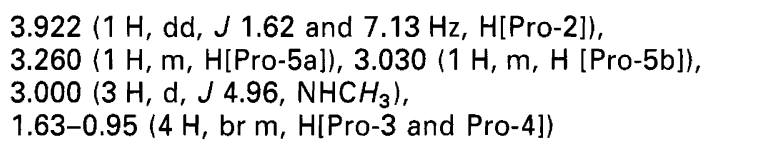 \\
\hline $2 j$ & $\begin{array}{l}445(M), 443\left(M-\mathrm{H}_{2}\right) \\
428\left(M-\mathrm{NH}_{3}\right), 398\left(443-\mathrm{NH}_{3}-\mathrm{CO}\right) \\
372\left(M-\mathrm{NH}_{2} \mathrm{CHCONH}_{2}\right) \\
130(M-\mathrm{TrtNHCHCONH})\end{array}$ & $\begin{array}{l}\text { 8.121 }(1 \mathrm{H}, \text { br s, CONH), } \\
7.52-7.02(18 \mathrm{H}, \mathrm{m}, \mathrm{PhH}, \mathrm{CONH}, \operatorname{ImH}), \\
6.913(1 \mathrm{H}, \mathrm{d}, J 2.02 \mathrm{~Hz}, \mathrm{ImH}), 3.546\left(1 \mathrm{H}, \mathrm{m},{ }^{*} \mathrm{CH}\right), \\
3.161(1 \mathrm{H}, \mathrm{dd}, J 5.82 \text { and } 14.36 \mathrm{~Hz}, \mathrm{ImCHa}), \\
2.911(1 \mathrm{H}, \mathrm{d}, J 5.59 \mathrm{~Hz} \text {, TrtNH), } \\
2.560(1 \mathrm{H}, \mathrm{dd}, J 6.12 \text { and } 14.36 \mathrm{~Hz}, \operatorname{ImCHb})\end{array}$ \\
\hline 2k & $\begin{array}{l}465(M+\mathrm{H}), 392\left(\mathrm{M}-\mathrm{CONMe}_{2}\right) \\
343\left(\mathrm{M}-\left(\mathrm{CH}_{2} \mathrm{O}+\mathrm{CH}_{2} \mathrm{Ph}\right)\right] \\
221\left(343-\mathrm{HNMe}_{2}-\mathrm{Ph}\right),(\mathrm{CONMe})\end{array}$ & $\begin{array}{l}4.490\left(2 \mathrm{H}, \mathrm{s}, \mathrm{CH}_{2} \mathrm{Ph}\right) \text {, } \\
3.976(1 \mathrm{H}, \mathrm{dd}, \mathrm{J} 8.70 \text { and } 5.30 \mathrm{OCHa} \text { or } \mathrm{OCHb}), \\
3.739\left(1 \mathrm{H}, \mathrm{m},{ }^{*} \mathrm{CH}\right), 3.563(1 \mathrm{H}, \mathrm{t}, \mathrm{J} 8.70 \mathrm{OCHa} \text { or } \mathrm{OCHb}) \text {, } \\
3.40(1 \mathrm{H}, \mathrm{br}, \mathrm{Tr} \mathrm{NH}), 2.440 \text { and } 2.426(2 \times 3 \mathrm{H}, \mathrm{s}, \mathrm{NMe})\end{array}$ \\
\hline
\end{tabular}




\begin{tabular}{|c|c|c|}
\hline Amide & MS data & NMR data (广், ppm) \\
\hline 21 & $\begin{array}{l}431(M+H), 398(M-M e O H) \\
369(398-E t), 84 \text { (COCHNEt) }\end{array}$ & $\begin{array}{l}5.924(1 \mathrm{H}, \text { br t, J } 5.85 \mathrm{~Hz}, \mathrm{CONH}), 3.647(3 \mathrm{H}, \mathrm{s}, \mathrm{OMe}), \\
3.223\left(1 \mathrm{H}, \mathrm{m},{ }^{*} \mathrm{CH}\right), 3.022(1 \mathrm{H}, \mathrm{d}, J 5.82 \mathrm{~Hz}, \mathrm{TrtNH}), \\
2.811\left(2 \mathrm{H}, \mathrm{dq}, J 5.85 \text { and } 7.27 \mathrm{~Hz}, \mathrm{NCH}_{2}\right), \\
2.52-2.15\left(2 \mathrm{H}, \mathrm{m}, \mathrm{CH}_{2} \mathrm{CO}_{2} \mathrm{Me}\right), \\
2.08-1.76\left(2 \mathrm{H}, \mathrm{m}, \mathrm{CHCH}_{2}\right), \\
0.879\left(3 \mathrm{H}, \mathrm{t}, J 7.27 \mathrm{~Hz}, \mathrm{CH}_{2} \mathrm{CH}_{3}\right),\end{array}$ \\
\hline $2 m$ & $\begin{array}{l}431(M+\mathrm{H}), 398(M-\mathrm{MeOH}) \\
358\left(M-\mathrm{CONMe}_{2}\right), 72(\mathrm{CONMe})\end{array}$ & $\begin{array}{l}3.686\left(3 \mathrm{H}, \mathrm{s}, \mathrm{CO}_{2} \mathrm{CH}_{3}\right), 3.360\left(1 \mathrm{H}, \mathrm{br},{ }^{*} \mathrm{CH}\right) \text {, } \\
2.500 \text { and } 2.396\left(2 \times 3 \mathrm{H}, \mathrm{s}, \mathrm{NCH}_{3}\right) \text {, } \\
\left.\text { 2.90-1.42(4 H, m, } \mathrm{CH}_{2} \mathrm{CH}_{2}\right)\end{array}$ \\
\hline
\end{tabular}

${ }^{a}$ All MS spectra showed strong peaks at $\mathrm{m} / 2243\left(\mathrm{Trt}^{+}\right.$) and 165 (fluorenyl cation) and weak peaks above 243 . However, TrtPhe- $\mathrm{NMe}_{2}$ showed the corresponding peaks at $\mathrm{m} / \mathrm{z} 242$ (Trt-H) and 166 (fluorenyl cation $+\mathrm{H}$ ). Only the diagnostically most significant peaks are tabulated. In general, all NMR spectra showed a complex multiplet in the region $7.10-7.40$ ppm due to the aromatic protons.

$\gamma$-ester function in the subsequent reduction of the $\alpha$-amide function. Further work on the preparation of a wider variety of diamines like $\mathbf{4}$ and their application in asymmetric synthesis is now in progress.

\section{Experimental}

General. The benzotriazolyl esters 1 used in the present work were obtained from the corresponding L-amino acids through $N$-tritylation followed by esterification with $\mathrm{HOBt}$ in the presence of $\mathrm{DCC}^{2}$ Capillary melting points were measured on a Büchi SMP-20 apparatus and are uncorrected. Optical rotations were determined with a Carl-Zeiss precision polarimeter. IR spectra were recorded for $\mathrm{KBr}$ pellets or neat samples (for oily compounds) on a Perkin-Elmer 16PC FT-IR spectrophotometer. ${ }^{1} \mathrm{H}$ NMR spectra were obtained at $200.13 \mathrm{MHz}$ on a Bruker AC-200 instrument using $\mathrm{CDCl}_{3}$ as the solvent and $\mathrm{Me}_{4} \mathrm{Si}$ as an internal standard. Mass spectra were obtained on a Fisons VG 7070E mass spectrometer at en electron bombardment energy of $70 \mathrm{eV}$, using the direct inlet probe at a temperature of $250{ }^{\circ} \mathrm{C}$. FAB mass spectra were recorded on a Fisons-VG ZAB $2 \mathrm{f}$ instrument, operated at $8 \mathrm{keV}$ accelerating potential, with an M-SCAN ion gun operated at $10 \mu \mathrm{A}$ and a $9 \mathrm{keV}$ xenon beam. The matrix was $m$-nitrobenzyl alcohol with the exception of tosylate $\mathbf{3 c}$ for which glycerol was used as the matrix. FCC was performed on Merck silica gel 60 (230-400 mesh) and TLC on Merck silica gel $F_{254}$ films $(0.2 \mathrm{~mm})$ precoated on aluminium foil. The solvent systems used were: (A) PhMe-AcOEt (8:2) and (B) $\mathrm{CHCl}_{3}-\mathrm{MeOH}(9: 1)$. Spots were visualized with UV light at $254 \mathrm{~nm}$ and with ninhydrin. THF was distilled from $\mathrm{Na}$-benzophenone.

Amidation procedure. To an ice-cold solution of esters 1 $(8 \mathrm{mmol})$ in DMF $(8 \mathrm{ml}), 12 \mathrm{mmol}$ each of $\mathrm{Et}_{3} \mathrm{~N}$ $(1.64 \mathrm{ml})$ and aq. amine were added and the resulting reaction mixture was stirred at $0{ }^{\circ} \mathrm{C}$ for $15-30 \mathrm{~min}$ and then diluted with $5 \%$ aq. citric acid. The precipitated product was extracted twice into EtOAc and the combined organic layers were washed sequentially with $\mathrm{H}_{2} \mathrm{O}$, $5 \%$ aq. $\mathrm{NaHCO}_{3}, \mathrm{H}_{2} \mathrm{O}$ and brine, and dried $\left(\mathrm{Na}_{2} \mathrm{SO}_{4}\right)$. Evaporation of the solvent and addition of $\mathrm{Et}_{2} \mathrm{O}$ or $\mathrm{Et}_{2} \mathrm{O}$-hexane $(1: 1)$ to the residue caused crystallization of the pure amides 2 . Yields, physical constants and spectral data are tabulated in Tables 1 and 2. In one of the preparations of amide $\mathbf{2 c}$, the reaction mixture was fractionated by FCC using solvent system $\mathrm{A}$ as the eluent, and the unchanged 'active' amide form $\left(R_{\mathrm{f}} 0.54\right)$ was isolated and characterised by IR (3332 and $\left.1726 \mathrm{~cm}^{-1}\right)$ and MS $(m / z): 475 \quad(M-\mathrm{H}), 433$ $\left(M-\mathrm{CHMe}_{2}\right), 399(M-\mathrm{Ph}), 314(M-\mathrm{COBtO})$ and 243 ( Trt).

Detritylation procedure. A suspension of amide $\mathbf{2}$ $(4 \mathrm{mmol})$ and $p$-toluenesulfonic acid hydrate $(1.1 \mathrm{~g}$, $6 \mathrm{mmol})$ in 2-propanol $(12 \mathrm{ml})$ was brought to reflux. The resulting solution was kept at reflux temperature for $5 \mathrm{~min}$ and then left to attain room temperature. Trituration with $\mathrm{Et}_{2} \mathrm{O}$ followed by refrigeration gave the corresponding crystalline tosylates 3 (Table 1). The FAB mass spectra $[\mathrm{m} / \mathrm{z}(\%$ rel. int. $)]$ for tosylates 3 were as follows. 3a: $205(10.5,[2 M+1]), 103(100,[M+1]), 46$ (23.7, $\left.\left[\mathrm{H}_{2} \mathrm{NCH}_{2} \mathrm{Me}+\mathrm{H}\right]\right)$. 3b: $179(100,[M+1]), 120$ (44.7, $\left.\left[\mathrm{PhCH}=\mathrm{CHNH}_{2}+\mathrm{H}\right]\right) .3 \mathrm{c}: 204(100,[M+1]), 130$ $\left(31,\left[\mathrm{C}_{9} \mathrm{H}_{8} \mathrm{~N}\right]\right)$.

Procedure for $\mathrm{LiAlH}_{4}$ reduction. To a suspension of $\mathrm{LiAlH}_{4}(0.46 \mathrm{~g}, 12 \mathrm{mmol})$ in refluxing THF $(4 \mathrm{ml})$ was added amide $2(4 \mathrm{mmol})$ in portions over a period of $30 \mathrm{~min}$ and the resulting reaction mixture was further refluxed for 1-2 days, with the exception of amide $\mathbf{2 m}$ for which only $1 \mathrm{~h}$ was required for completion of the reduction. The reaction mixture was then cooled to 0 $\mathrm{C}$ and the excess $\mathrm{LiAlH}_{4}$ was destroyed by the dropwise addition of a saturated aq. solution of $\mathrm{Na}_{2} \mathrm{SO}_{4}$. The resulting mixture was filtered and the filtrate evaporated to dryness. The residue was partitioned between EtOAc and brine and the organic phase was dried $\left(\mathrm{Na}_{2} \mathrm{SO}_{4}\right)$ 
and evaporated to dryness. The residue was subjected to FCC with solvent system $B$ as the eluent to give pure diamines 4 (Table 1$)$. The FAB mass spectra $[\mathrm{m} / z$ ( $\%$ rel. int.)] for diamines $\mathbf{4}$ were as follows for $\mathbf{4 a}$ : 331 $(100[M+1]), 286 \quad(9, \quad[\operatorname{TrtNH}=\mathrm{CHMe}]), \quad \mathbf{4 b}: 357$ $(100, \quad[M+1]) . \quad 4 c: 407 \quad(85.7, \quad[M+1]), \quad 363$ $\left(100,\left[\mathrm{TrtNH}=\mathrm{CHCH}_{2} \mathrm{Ph}\right]\right) . \quad 4 d: 389(100,[M+1])$, $330 \quad\left(26.7, \quad\left[\mathrm{TrtNH}=\mathrm{CH}\left(\mathrm{CH}_{2}\right)_{3} \mathrm{OH}\right]\right), \quad 311 \quad$ (26.7, $\left[\mathrm{TrtNHCH}=\mathrm{CHCH}=\mathrm{CH}_{2}\right]$ ).

\section{References}

1. Somlai, C., Szokan, G. and Penke, B. Synthesis (1995) 683 and references cited therein.

2. Barlos, K., Papaioannou, D. and Theodoropoulos, D. Int. J. Peptide Protein Res. 23 (1984) 300.

3. Barlos, K., Papaioannou, D. Patrianakou S. and Tsegenidis, T. Liebigs Ann. Chem. (1986) 1950.

4. Barlos, K., Papaioannou, D. and Sanida, C. Liebigs Ann. Chem. (1984) 1308.

5. Chen, S.-T., Wu, S.-H. and Wang, K.-T. Synthesis (1989) 37.

6. Mamos, P., Karigiannis, G., Athanassopoulos, C., Bichta, S. Kalpaxis, D., Papaioannou, D. and Sindona, G. Tetrahedron Lett. (1995) 5187 and references cited therein. 